\title{
Combining Independent Tests of Conditional Shifted Exponential Distribution
}

\author{
Abedel-Qader S. Al-Masri \\ Yarmouk University, Irbid, Jordan, almasri68@yu.edu.jo
}

Follow this and additional works at: http:// digitalcommons.wayne.edu/jmasm

Part of the Applied Statistics Commons, Social and Behavioral Sciences Commons, and the $\underline{\text { Statistical Theory Commons }}$

\section{Recommended Citation}

Al-Masri, Abedel-Qader S. (2010) "Combining Independent Tests of Conditional Shifted Exponential Distribution," Journal of Modern Applied Statistical Methods: Vol. 9 : Iss. 1 , Article 21.

DOI: $10.22237 /$ jmasm/1272687600

Available at: http://digitalcommons.wayne.edu/jmasm/vol9/iss1/21

This Regular Article is brought to you for free and open access by the Open Access Journals at DigitalCommons@WayneState. It has been accepted for inclusion in Journal of Modern Applied Statistical Methods by an authorized editor of DigitalCommons@WayneState. 


\title{
Combining Independent Tests of Conditional Shifted Exponential Distribution
}

\author{
Abedel-Qader S. Al-Masri \\ Yarmouk University, \\ Irbid, Jordan
}

The problem of combining $\mathrm{n}$ independent tests as $n \rightarrow \infty$ for testing that variables are uniformly distributed over the interval $(0,1)$ compared to their having a conditional shifted exponential distribution with probability density function $f(x \mid \theta)=e^{-(x-\gamma \theta)}, \quad x \geq \gamma \theta, \quad \theta \in[a, \infty), a \geq 0$ was studied. This was examined for the case where $\theta_{1}, \theta_{2}, \ldots$ are distributed according to the distribution function (DF) $\mathrm{F}$ and when the DF is Gamma $(1,2)$. Six omnibus methods were compared via the Bahadur efficiency. It is shown that, as $\gamma \rightarrow 0$ and $\gamma \rightarrow \infty$, the inverse normal method is the best among the methods studied.

Key words: Conditional shifted exponential, combining independent tests, omnibus methods, Bahadur efficiency.

Introduction

The combination of independent tests of hypothesis is an important and a popular statistical practice. Many methods are available to use to combine independent tests; these methods are compared by using different criteria including Exact Bahadur Slope (EBS), Approximate Bahadur Slope (ABS), Pitman Efficiency, Local Power, Admissibility and others.

If $\mathrm{H}_{0}$ is a simple hypothesis, Birnbaum (1955) showed that, for given any nonparametric combination method with a monotone increasing acceptance region, there exists a problem for which this method is most powerful against some alternative. Littell and Folks (1971) studied four methods of combining a finite number of independent tests. They found that the Fisher method is better than the inverse normal method, the minimum of $\mathrm{p}$-value method and maximum of $\mathrm{p}$-vales via Bahadur efficiency. Later, Littell and Folks (1973) showed under mild conditions that the Fisher's method is

Abedel-Qader S. Al-Masri is an instructor in the Department of Statistics. Email him at: almasri68@yu.edu.jo. optimal among all methods for combining a finite number of independent tests. Brown, Cohen and Strawderman (1976) have shown that such tests form a complete class.

The Specific Problem

Consider $\mathrm{n}$ hypotheses of the form:

$$
\begin{gathered}
H_{o}^{(i)}: \eta_{i}=\eta_{o}^{i} \\
\text { vs } \\
H_{1}^{(i)}: \eta_{i} \in \Omega_{i}-\left\{\eta_{o}^{i}\right\}
\end{gathered}
$$

such that $H_{0}^{(i)}$ is rejected for large values, $\mathrm{i}=1$, $2, \ldots, \mathrm{n}$ of some continuous random variable $\mathrm{T}^{(\mathrm{i})}$. The $\mathrm{n}$ hypotheses are combined into one as

$$
\begin{gathered}
H_{0}:\left(\eta_{1}, \ldots, \eta_{n}\right)=\left(\eta_{o}^{1}, \ldots, \eta_{o}^{n}\right) \\
H_{1}^{(i)}:\left(\eta_{1}, \ldots, \eta_{n}\right) \in\left\{\prod_{i=1}^{n} \Omega_{i}-\left\{\left(\eta_{o}^{1}, \ldots, \eta_{o}^{n}\right)\right\}\right\} .
\end{gathered}
$$

For $\mathrm{i}=1,2, \ldots, \mathrm{n}$ the $\mathrm{p}$-value of the $\mathrm{i}^{\text {th }}$ test is given by

$$
P_{i}(t)=P_{H_{o}^{(i)}}\left(T^{(i)}>t\right)=1-F^{i}(t)
$$




\section{COMBINING INDEPENDENT TESTS OF EXPONENTIAL DISTRIBUTION}

where $\mathrm{F}^{\mathrm{i}}$ is the $\mathrm{DF}$ of $\mathrm{T}^{(\mathrm{i})}$ under $H_{o}^{(i)}$. Note that under $H_{o}^{(i)}$ the random variable $\mathrm{P}_{\mathrm{i}} \sim \mathrm{U}(0,1)$ under $H_{1}^{(i)}$ has some distribution that is not $\mathrm{U}(0,1)$.

If considering the special case where $\eta_{\mathrm{i}}$ $=\theta$ and $\eta_{o}^{i}=\theta_{o}$ for $\mathrm{i}=1, \ldots, \mathrm{n}$, and also assume that $\mathrm{T}^{(1)}, \ldots, \mathrm{T}^{(\mathrm{n})}$ are independent, then (1) reduces to

$$
\begin{gathered}
H_{o}: \theta=\theta_{o} \\
\text { vs } \\
H_{1}: \theta \in \Omega-\left\{\theta_{o}\right\} .
\end{gathered}
$$

It follows that the $\mathrm{p}$-values $\mathrm{P}_{1}, \ldots, \mathrm{P}_{\mathrm{n}}$ are also independent identically distributed random variables that have a $\mathrm{U}(0,1)$ distribution under $\mathrm{H}_{0}$, and under $\mathrm{H}_{1}$ have a distribution whose support is a subset of the interval $(0,1)$ and is not a $\mathrm{U}(0,1)$ distribution. Therefore, if $\mathrm{f}$ is the probability density function (pdf) of $\mathrm{P}$, then (4) is equivalent to

$$
\begin{gathered}
\mathrm{H}_{0}: \mathrm{P} \sim \mathrm{U}(0,1) \\
\text { vs } \\
\mathrm{H}_{1}: P+U(0,1)
\end{gathered}
$$

where $P$ has a pdf $\mathrm{f}$ with support a subset of the interval $(0,1)$.

This study considers the case: $\eta_{\mathrm{i}}=\gamma \theta_{\mathrm{i}}, \mathrm{i}$ $=1, \ldots, \mathrm{n}$, where $\theta_{1}, \ldots, \theta_{\mathrm{n}}$ are independent identically distributed with DF F with support $[a, \infty), a \geq 0$ and the following hypothesis is tested:

$$
\begin{gathered}
H_{o}: \gamma=0 \\
\text { vs } \\
H_{1}: \gamma>0
\end{gathered}
$$

where the $i^{\text {th }}$ problem is based on $T_{1}, \ldots, T_{n}$, which are independent random variables from a conditional shifted exponential with pdf $f(x \mid \theta)=e^{-(x-\gamma \theta)}, x \geq \gamma \theta$ and $\theta_{1}, \ldots, \theta_{\mathrm{n}}$ are independent identically distributed with DF $\mathrm{F}$ with support $[a, \infty), a \geq 0$. Six methods are compared, namely: maximum of p-values method, Tippett's method, Fisher method, logistic method, inverse normal method, and the sum of p-values method. These methods reject $\mathrm{H}_{\mathrm{o}}$ for large values of

$$
\begin{gathered}
-\max _{1 \leq i \leq n}\left(p_{i}\right) \text { (Maximum of } \mathrm{p} \text {-values), } \\
-\min _{1 \leq i \leq n}\left(p_{i}\right) \text { (Tippett's), } \\
-2 \sum_{i=1}^{n} \frac{\ln P_{i}}{\sqrt{n}} \text { (Fisher), } \\
-\sum_{i=1}^{n} \ln \left(\frac{P_{i}}{1-P_{i}}\right) / \sqrt{n} \text { (Logistic), } \\
-\sum_{i=1}^{n} \frac{\Phi^{-1}\left(P_{i}\right)}{\sqrt{n}} \text { (Inverse normal) }
\end{gathered}
$$

and

$$
-\sum_{i=1}^{n} \frac{P_{i}}{\sqrt{n}} \text { (Sum of p-values). }
$$

Derivation of EBS

Let $X_{1}, \ldots, X_{n}$ be an independent identically distributed pdf with $\mathrm{f}(\mathrm{x}, \theta)$, the hypotheses test hypotheses are $\mathrm{H}_{0}: \theta=\theta_{0}$ vs. $\mathrm{H}_{1}$ : $\theta \in \Omega-\left\{\theta_{0}\right\},\left\{T_{n}^{1}\right\}$ and $\left\{T_{n}^{2}\right\}$ are two sequences of test statistics for testing $\mathrm{H}_{\mathrm{o}}$, and the p-value of $T_{n}^{i} \quad$ is $\quad P_{n}^{i}=1-F_{i}\left(T_{n}^{i}\right) \quad$ where $F_{i}(t)=P_{H_{o}}\left(T_{n}^{i} \leq t_{i}\right)$ for $\mathrm{i}=1,2$.

Under these assumptions, there usually exists a positive valued function $\mathrm{C}_{\mathrm{i}}(\theta)$, which is termed the EBS of the sequence $\left\{T_{n}^{i}\right\}$ at $\theta$. This EBS sequence has the property that $\mathrm{C}_{\mathrm{i}}(\theta)=\lim _{n \rightarrow \infty}-\frac{2}{n} \ln P_{n}^{i}$ w. p. 1 under $\theta$, and the Bahadur efficiency of $\left\{T_{n}^{1}\right\}$ relative to $\left\{T_{n}^{2}\right\}$ which is given by $\frac{C_{1}(\theta)}{C_{2}(\theta)}$. Therefore, comparing two tests via the Bahadur efficiency is equivalent to comparing their corresponding EBS's. The following three theorems provide the EBS for tests based on sums of independent identically distributed random variables. 
Theorem 1

Let $X_{1}, \ldots, \quad X_{n}$ be independent identically distributed random variables with $\mathrm{DF}$ $\mathrm{F}$ and $S_{n}=\sum_{i=1}^{n} X_{i}$. Assume that the moment generating function $M(z)=E_{F} e^{z X}$ exists and is near zero. If $m(t)=\underset{z}{\inf } e^{-z t} M(z)$, then $\lim _{n \rightarrow \infty}-\frac{2}{n} \ln P_{F}\left[S_{n} \geq n t\right]=-2 \ln m(t)$.

\section{Theorem 2}

Let $\left\{\mathrm{T}_{\mathrm{n}}\right\}$ be a sequence of test statistics satisfying the following conditions:

1. Under $\mathrm{H}_{1}: \frac{T_{n}}{\sqrt{n}} \rightarrow b(\theta)$ w.p.1 under $\theta$ where $b(\theta)$ is a real function.

2. An open interval I containing $\{b(\theta): \theta \in \Omega\}$ exists and a function $\mathrm{g}$ continuous on $\mathrm{I}$ such that $\lim _{n \rightarrow \infty}-\frac{2}{n} \ln \left[1-F_{n}(t \sqrt{n})\right]=g(t)$ where $F_{n}$ is the DF of $T_{n}$ under $H_{0}$.

Thus the EBS of $\left\{\mathrm{T}_{\mathrm{n}}\right\}$ is $\mathrm{C}(\theta)=\mathrm{g}(\mathrm{b}(\theta))$.

\section{Theorem 3}

Let $U_{1}, U_{2}, \ldots$ be independent identically distributed random variables. To test $\mathrm{H}_{\mathrm{o}}: \mathrm{U}_{\mathrm{i}} \sim \mathrm{U}(0,1)$ vs $\mathrm{H}_{1}: \mathrm{U}_{\mathrm{i}} \sim \mathrm{f}$ on $(0,1)$, which is not $\mathrm{U}(0,1)$, then

1. $C_{\max }(f)=-2 \ln \left(\operatorname{ess.Sup}_{f}(u)\right)$, where ess. $\operatorname{Sup}_{\mathrm{f}}(\mathrm{u})=\operatorname{Sup}\{\mathrm{u}: \mathrm{f}(\mathrm{u})>0\}$ w.p. 1 under f.

2. If $t(\ln t)^{2} f(t) \rightarrow 0$ as $t \rightarrow 0$, then $\mathrm{C}_{\min }(\mathrm{f})=0$.

Note that for testing problem (6), the $i^{\text {th }}$ p-value is:

$$
P_{i}=P\left(X \geq x_{i}\right)=e^{-x_{i}}
$$

The next four lemmas give the EBS for Fisher $\left(C_{F}\right)$, logistic $\left(C_{L}\right)$, inverse normal $\left(C_{N}\right)$, sum of p-values $\left(\mathrm{C}_{\mathrm{S}}\right)$, Tippett's $\left(\mathrm{C}_{\mathrm{T}}\right)$ and maximum of $\mathrm{p}$ values $\left(C_{\max }\right)$ methods.

\section{Lemma 1}

$$
\mathrm{C}_{\mathrm{F}}(\gamma)=2 \gamma \mathrm{E}_{\mathrm{F}} \theta-2 \ln \left(1+\gamma \mathrm{E}_{\mathrm{F}} \theta\right)
$$

$$
\mathrm{C}_{\mathrm{S}}(\gamma)=-2 \ln m_{S}\left(\frac{1}{2} E_{F}\left(e^{-\gamma \theta}\right)\right),
$$

where

$$
\begin{aligned}
& m_{S}(t)=\inf _{z>0}\left\{e^{-t z} \frac{1-e^{-z}}{z}\right\} \\
& \mathrm{C}_{\mathrm{L}}(\gamma)=-2 \ln m_{L}\left(b_{L}(\gamma)\right),
\end{aligned}
$$

where

$$
m_{L}(\gamma)=\inf _{0<z<1}\left\{e^{-b_{L}(\gamma) z} \pi z \operatorname{CSC}(\pi z)\right\}
$$

and

$$
\begin{gathered}
b_{L}(\gamma)=\gamma \mathrm{E}_{\mathrm{F}} \theta-\mathrm{E}_{\mathrm{F}}\left(e^{\gamma \theta}-1\right) \ln \left(1-e^{-\gamma \theta}\right) \\
C_{N}(\gamma)=\left(E_{F}\left\{e^{\gamma \theta} \varphi\left(\Phi^{-1}\left(e^{\gamma \theta}\right)\right)\right\}\right)^{2}
\end{gathered}
$$

The proof for Lemma 1 follows from Theorems (1) and (2).

Lemma 2

Let $\mathrm{U}_{1}, \ldots, \mathrm{U}_{\mathrm{n}}$ be independent identically distributed like $U$ with pdf $f$, if the test hypotheses are:

$$
\begin{gathered}
\mathrm{H}_{\mathrm{o}}: \mathrm{U}_{\mathrm{i}} \sim \mathrm{U}(0,1) \\
\text { vs }
\end{gathered}
$$

$$
\mathrm{H}_{1}: U_{i} \sim f \text { on }(0,1) \text { but } \operatorname{not} \mathrm{U}(0,1) \text {, }
$$

then

$$
\mathrm{C}_{\max }(\mathrm{f})=-2 \ln \left(\text { ess. } \sup _{f}(U)\right)
$$

Where ess. $\operatorname{Sup}_{\mathrm{f}} \mathrm{U}=\operatorname{Sup}\{\mathrm{u}: \mathrm{f}(\mathrm{u})>0\}$ w.p.1 under $\mathrm{f}$.

\section{Proof: Lemma 2} $\mathrm{H}_{\mathrm{o}}$. Then for

Let $G_{0}(t)$ be the DF of $-\max _{i} U_{i}$ under

$$
-1<\mathrm{t}<0,1-\mathrm{G}_{\mathrm{o}}(\mathrm{t})=(-\mathrm{t})^{\mathrm{n}},
$$

which implies that 
$-2 / n \ln \left[1-G_{o}\left(-\max _{i} U_{i}\right)\right]=-2 \ln \max _{i} U_{i}$

Therefore,

$$
\begin{aligned}
\mathrm{C}_{\max }(\mathrm{f}) & =\lim _{n \rightarrow \infty}\left[-2 \ln \max _{i} U_{i}\right] \\
& =-2 \ln \lim _{n \rightarrow \infty} \max _{i} U_{i} \\
& =-2 \ln \left(\operatorname{ess} \sup _{f} U\right) .
\end{aligned}
$$

Lemma 3

$$
\mathrm{C}_{\max }(\gamma)=2 \gamma \mathrm{a}
$$

Proof: Lemma 3

Assume $g(\theta)$ is the pdf of the DF F, then the joint pdf of $\mathrm{x}$ and $\theta$ is

$$
\mathrm{h}(\mathrm{x}, \theta)=g(\theta) f(x \mid \theta),
$$

where $\mathrm{f}(\mathrm{x} \mid \theta)=e^{-(x-\gamma \theta)}, x>\gamma \theta$. Then the marginal pdf of $x$ is

$$
\begin{aligned}
f(x) & =\int_{a}^{x / \gamma} h(x, \theta) d \theta \\
& =\int_{a}^{x / \gamma} e^{-(x-\gamma \theta)} g(\theta) d \theta, \quad x>a \gamma, a \geq 0 \\
& =e^{-x} \int_{a}^{x / \gamma} e^{\gamma \theta} d F(\theta), \quad x>\gamma \theta
\end{aligned}
$$

The under $\gamma$ the $\mathrm{p}$-value is $e^{-x} \equiv \mathrm{P}$ satisfies $0<P<e^{-\gamma a}$, then ess.sup $\mathrm{P}=e^{-\gamma a}$, which implies $C_{\max }(\gamma)=2 \gamma$ a by theorem (3).

Lemma 4

$$
\mathrm{C}_{\mathrm{T}}(\gamma)=0
$$

Proof: Lemma 4

$$
g(p)=\int_{a}^{-\ln p / \gamma} e^{\gamma \theta} g(\theta) d \theta
$$

$$
\begin{aligned}
\lim _{p \rightarrow 0} p & (\ln p)^{2} g(p) \\
& =\lim _{p \rightarrow 0} \frac{(\ln p)^{2}}{1 / p} \int_{a}^{-\ln p / \gamma} e^{\gamma \theta} g(\theta) d \theta \\
& =\lim _{p \rightarrow 0}-p^{2}\left[\begin{array}{l}
\left.\frac{2 \ln p}{p} \int_{a}^{-\ln p / \gamma} e^{\gamma \theta} g(\theta) d \theta\right] \\
+\frac{(\ln p)^{2}}{p} g\left(\frac{-\ln p}{\gamma}\right)
\end{array}\right] \\
& =\lim _{p \rightarrow 0}-2 p \ln p \int_{a}^{-\ln p / \gamma} e^{\gamma \theta} g(\theta) d \theta \\
& =0
\end{aligned}
$$

using L'hopital rule because $\mathrm{g}(\infty)=0$ and $\lim _{p \rightarrow 0} p(\ln p)^{2}=0$.

\section{Results}

First, it the limits of the ratio of any two methods under study were found as $\gamma \rightarrow 0$ and $\gamma \rightarrow \infty$. This gives the following results.

Corollary 1

$$
\lim _{\gamma \rightarrow 0} \frac{C_{\max }(\gamma)}{C_{a}(\gamma)}=0
$$

where

$$
C_{a}(\gamma)=\left\{C_{S}(\gamma), C_{N}(\gamma), C_{L}(\gamma), C_{F}(\gamma)\right\}
$$

and

$$
\lim _{\gamma \rightarrow 0} \frac{C_{F}(\gamma)}{C_{S}(\gamma)}=\frac{1}{3},
$$

$$
\begin{aligned}
\lim _{\gamma \rightarrow 0} \frac{C_{S}(\gamma)}{C_{L}(\gamma)} & =\lim _{\gamma \rightarrow 0} \frac{C_{S}(\gamma)}{C_{N}(\gamma)} \\
& =\lim _{\gamma \rightarrow 0} \frac{C_{F}(\gamma)}{C_{L}(\gamma)} \\
& =\lim _{\gamma \rightarrow 0} \frac{C_{F}(\gamma)}{C_{N}(\gamma)} \\
& =\lim _{\gamma \rightarrow 0} \frac{C_{L}(\gamma)}{C_{N}(\gamma)} \\
& =0 .
\end{aligned}
$$




\section{AL-MASRI}

Corollary 2

$$
\lim _{\gamma \rightarrow \infty} \frac{C_{L}(\gamma)}{C_{F}(\gamma)}=1, \lim _{\gamma \rightarrow \infty} \frac{C_{F}(\gamma)}{C_{N}(\gamma)}=\lim _{\gamma \rightarrow \infty} \frac{C_{L}(\gamma)}{C_{N}(\gamma)}=0
$$

Proof 1

$$
\text { By (8) and (10) }
$$

$$
\frac{C_{L}(\gamma)}{C_{F}(\gamma)} \leq \frac{-2 \ln 2-2 \ln b_{L}(\gamma)+2 b_{L}(\gamma)}{2 \gamma E_{F} \theta-2 \ln \left(1+2 \gamma E_{F} \theta\right)},
$$

and

$$
\begin{aligned}
\lim _{\gamma \rightarrow \infty} \frac{C_{L}(\gamma)}{C_{F}(\gamma)} \leq & \lim _{\gamma \rightarrow \infty} \frac{-2 \ln 2-2 \ln b_{L}(\gamma)+2 b_{L}(\gamma)}{2 \gamma E_{F} \theta-2 \ln \left(1+2 \gamma E_{F} \theta\right)} \\
& =\lim _{\gamma \rightarrow \infty} \frac{-2 \frac{b_{L}^{\prime}(\gamma)}{b_{L}(\gamma)}+2 b_{L}^{\prime}(\gamma)}{2 E_{F} \theta-2 \frac{E_{F} \theta}{1+2 \gamma E_{F} \theta}} \\
& =1
\end{aligned}
$$

by using the L'Hopital rule.

Similarly, it can be shown that

$$
\lim _{\gamma \rightarrow \infty} \frac{C_{L}(\gamma)}{C_{F}(\gamma)} \geq 1
$$

Regarding $\mathrm{C}_{\mathrm{S}}(\gamma)$, nothing can be concluded about general prior F because $\lim _{\gamma \rightarrow \infty} \frac{b_{S}^{\prime}(\gamma)}{b_{S}(\gamma)}$ has an indeterminate for $(0 / 0)$, thus, only a certain prior - namely, $\mathrm{G}(\alpha, \beta)$ with $\alpha=1$ and $\beta=2$, is considered:

$$
\lim _{\gamma \rightarrow \infty} \frac{C_{S}(\gamma)}{C_{F}(\gamma)}=\lim _{\gamma \rightarrow \infty} \frac{-\frac{b_{S}^{\prime}(\gamma)}{b_{S}(\gamma)}}{E_{F} \theta} .
$$

Proof 2

$$
\text { By (8) and (10) }
$$

$$
\begin{gathered}
\lim _{\gamma \rightarrow \infty} \frac{C_{S}(\gamma)}{C_{F}(\gamma)} \leq \lim _{\gamma \rightarrow \infty} \frac{-2 \ln 2-2 \ln b_{S}(\gamma)}{2 \gamma E_{F} \theta-2 \ln \left(1+\lambda E_{F} \theta\right)} \\
=\lim _{\gamma \rightarrow \infty} \frac{-2 \frac{b_{S}^{\prime}(\gamma)}{b_{S}(\gamma)}}{2 E_{F} \theta-2 \frac{E_{F} \theta}{1+\gamma E_{F} \theta}} \\
=\lim _{\gamma \rightarrow \infty} \frac{-\frac{b_{S}^{\prime}(\gamma)}{b_{S}(\gamma)}}{E_{F} \theta}
\end{gathered}
$$

Similarly,

$$
\lim _{\gamma \rightarrow \infty} \frac{C_{S}(\gamma)}{C_{F}(\gamma)} \geq \lim _{\gamma \rightarrow \infty} \frac{-\frac{b_{S}^{\prime}(\gamma)}{b_{S}(\gamma)}}{E_{F} \theta},
$$

hence,

$$
\lim _{\gamma \rightarrow \infty} \frac{C_{L}(\gamma)}{C_{F}(\gamma)}=1
$$

From the above relations it may be concluded that locally as $\gamma \rightarrow 0$

$$
\mathrm{C}_{\mathrm{N}}(\gamma)>\mathrm{C}_{\mathrm{L}}(\gamma)>\mathrm{C}_{\mathrm{S}}(\gamma)>\mathrm{C}_{\mathrm{F}}(\gamma)>\mathrm{C}_{\max }(\gamma)>\mathrm{C}_{\mathrm{T}}(\gamma),
$$

but as $\gamma \rightarrow \infty$

$$
\mathrm{C}_{\mathrm{N}}(\gamma)>\mathrm{C}_{\mathrm{L}}(\gamma)>\mathrm{C}_{\max }(\gamma)>\mathrm{C}_{\mathrm{F}}(\gamma)>\mathrm{C}_{\mathrm{T}}(\gamma),
$$

The dominance of one method over the other in case $\gamma>0$ can be shown mathematically only in some cases. The proof is omitted because, although it is straightforward, it is lengthy; however, numerical comparison for all methods is shown in Table 1. It appears from Table 1 that

$$
\begin{gathered}
\gamma=0.05: \mathrm{C}_{\mathrm{N}}(\gamma)>\mathrm{C}_{\mathrm{L}}(\gamma)>\mathrm{C}_{\mathrm{S}}(\gamma)>\mathrm{C}_{\mathrm{F}}(\gamma) ; \\
\gamma \in[0.1,0.5]: \mathrm{C}_{\mathrm{S}}(\gamma)>\mathrm{C}_{\mathrm{N}}(\gamma)>\mathrm{C}_{\mathrm{L}}(\gamma)>\mathrm{C}_{\mathrm{F}}(\gamma) ; \\
\gamma=1.00: \mathrm{C}_{\mathrm{N}}(\gamma)>\mathrm{C}_{\mathrm{L}}(\gamma)>\mathrm{C}_{\mathrm{F}}(\gamma)>\mathrm{C}_{\mathrm{S}}(\gamma) ; \\
\gamma \in[2,3]: \mathrm{C}_{\mathrm{L}}(\gamma)>\mathrm{C}_{\mathrm{F}}(\gamma)>\mathrm{C}_{\mathrm{N}}(\gamma)>\mathrm{C}_{\mathrm{S}}(\gamma) ; \\
\gamma \in[5,8]: \mathrm{C}_{\mathrm{F}}(\gamma)>\mathrm{C}_{\mathrm{L}}(\gamma)>\mathrm{C}_{\mathrm{N}}(\gamma)>\mathrm{C}_{\mathrm{S}}(\gamma) ; \\
\gamma \in[10,20]: \mathrm{C}_{\mathrm{N}}(\gamma)>\mathrm{C}_{\mathrm{F}}(\gamma)>\mathrm{C}_{\mathrm{L}}(\gamma)>\mathrm{C}_{\mathrm{S}}(\gamma)
\end{gathered}
$$




\section{COMBINING INDEPENDENT TESTS OF EXPONENTIAL DISTRIBUTION}

Table 1: The Exact Bahadur Slopes for Conditional Shifted

Exponential with Prior $\mathrm{G}(1,2)$

\begin{tabular}{|c|c|c|c|c|}
\hline$\gamma$ & $\mathrm{C}_{\mathrm{S}}(\gamma)$ & $\mathrm{C}_{\mathrm{L}}(\gamma)$ & $\mathrm{C}_{\mathrm{F}}(\gamma)$ & $\mathrm{C}_{\mathrm{N}}(\gamma)$ \\
\hline 0.050 & 0.0249 & 0.0298 & 0.0094 & 0.0320 \\
\hline 0.100 & 0.0903 & 0.0818 & 0.0354 & 0.8447 \\
\hline 0.200 & 0.2512 & 0.2168 & 0.1271 & 0.2323 \\
\hline 0.300 & 0.4414 & 0.3796 & 0.2599 & 0.4096 \\
\hline 0.400 & 0.6329 & 0.5633 & 0.4244 & 0.6059 \\
\hline 0.500 & 0.8173 & 0.7644 & 0.6137 & 0.8153 \\
\hline 1.000 & 1.5887 & 1.9598 & 1.8028 & 1.9829 \\
\hline 2.000 & 2.6053 & 4.9002 & 4.7811 & 4.5961 \\
\hline 3.000 & 3.2781 & 8.1865 & 8.1082 & 7.3718 \\
\hline 5.000 & 4.1821 & 15.1632 & 15.2042 & 12.6267 \\
\hline 8.000 & 5.0527 & 26.0621 & 26.3336 & 21.1932 \\
\hline 10.00 & 5.4753 & 33.4909 & 33.9110 & 40.3568 \\
\hline 20.00 & 6.8134 & 71.6401 & 72.5729 & 162.3284 \\
\hline
\end{tabular}

References

Al-Masri, A.-Q. (1993). Combining independent tests in case of triangular and conditional shifted exponential distributions. Master of Science Thesis. Yarmouk University. Jordan.

Bahadur, R. R. (1959). Stochastic comparison of tests. Annals of Mathematical Statistics, 31, 276-292.

Birnbaum, A. (1955). Combining independent test of significance. Journal of the American Statistical Association, 49, 559-579.
Littell, R. C., \& Folks, L. J. (1971). Asymptotic optimality of Fisher's method of combining independent tests. Journal of the American Statistical Association, 66, 802-806.

Littell, R. C., \& Folks, L. J. (1973). Asymptotic optimality of Fisher's method of combining independent tests II. Journal of the American Statistical Association, 68, 193-194.

Serfling, R. J. (1980). Approximation theorems of mathematical statistics. New York: John Wiley. 